\title{
Optimal Homotopy Asymptotic and Homotopy Perturbation Methods for Linear Mixed Volterra-Fredholm Integral Equations
}

\author{
Hamed Daei Kasmaei ${ }^{1}$, Jalil Rashidinia ${ }^{1, *}$ \\ ${ }^{1}$ Department of Mathematics and Statistics, Faculty of Science, Central Tehran Branch, Islamic Azad \\ University, Tehran, Iran.
}

\section{Abstract}

In this paper, we study the mixed Volterra-Fredholm integral equations of the second kind by means of optimal homotopy asymptotic method (OHAM) and Homotopy Perturbation method (HPM).Our approach is independent of time and contains simple computations with quite acceptable approximate solutions in which approximate solutions obtained by these methods are close to exact solutions. Comparison of these methods have been discussed. The accuracy and efficiency of OHAM approach with respect to Homotopy Perturbation method (HPM) is illustrated by presenting four test examples. The results indicate that the OHAM is very effective and flexible to use with respect to HPM.

Keywords: Mixed Volterra-Fredholm integral equations, Optimal homotopy asymptotic method, Least square method, Homotopy Perturbation method.

\section{Lineer Karma Volterra-Fredholm İntegral Denklemleri için Optimal Homotopi Asimptotik ve Homotopi Perturbasyon Metotları}

Öz

$\mathrm{Bu}$ çalışmada, karma Volterra-Fredholm integral denklemleri optimal homotopi asimptotik metod (OHAM) ve Homotopi Perturbasyon metodu (HPM) vasıtasıyla irdelenmiştir. Yaklaşımımız zamandan bağımsız ve basit hesaplamalar yolu ile tam çözüme oldukça yaklaşık çözümler veren bir yöntemdir. Bu iki yöntemin karşılaştırılması tartışılmıştır. OHAM yaklaşımının doğruluğu ve etkinliği HPM çözümleri ile dört örnek kullanılarak karşılaştırılmıştır. Sonuçlar OHAM in HPM ye göre daha verimli ve esnek bir yöntem olduğunu göstermektedir.

Anahtar Kelimeler: Karma Volterra-Fredholm integral denklemleri, Optimal homotopi asimptotik metodu, En küçük kareler yöntemi, Homotopi Perturbasyon Metodu.

\footnotetext{
*e-mail: rashidinia@iust.ac.ir
} 


\section{Introduction}

Many models in different areas of applied mathematics lead to mixed Volterra-Fredholm integral equations. They have been studied in some cases such as boundary value problems of prabolic type, Spatio-Temporal problem in Epidemic development and some issues in different physical and biological structures. Spatial-temporal problems are studied a lot in statistical mathematics.They can be difined for example in a monitoring network of an atmospheric pollutant or a network in meteorological problems so that their data are analyzed at regular intervals. Epidemic data have Spatio-temporal processes with autoregressive nature.Spread of a communicable disease in a population is described by epidemic structures. Spatio Temporal analysis has been studied by many researchers [5-7, 11, 18, 37, 43].

Therefore, numerical, analytical and semi-analytic methods play an important role to analyze such these phenomena. Spatio-temporal analysis in some mathematical models leads to mixed VolterraFredholm integral equations. A comprehensive description for formulation of these models has proposed by Wazwaz [45] and other researchers in this field [3-4 , 44, 47-49]. The Volterra-Fredholm integral equations of mixed form [45] is in the folowing format :

$$
v(x)=f(x)+\int_{c}^{x} \int_{c}^{d} K(y, z) v(z) d z d y
$$

Such that $f(x)$ and $K(y, z)$ can be defined as known functions. It is noticed that Eq.1 includes mixed Volterra and Fredholm integral equations. Fredholm integral is defined in the interior part and Volterra integral is appeared in exterior one. Furthermore, the unknown function $v(x)$ exists in inside and outside of integral part that is called characteristic feature for these types of equations. The exact and analytic solutions of mentioned equations cannot be found easily. Hence some numerical methods have been suggested to solve them [2, 8, 13, 15, 24, 28,31, 36, 47, 48]. In some cases , partial differential equations in physics can be converted to mixed Volterra-Fredholm integral equation [32]. Double integral equations have been solved through methods like Collocation and Galerkin method, Spline functions and Taylor series expansion method [1, 3, 32, 44].

Now, we explain optimal homotopy asymptotic method (OHAM ) to solve these types of equations in comparison with HPM. Marinca and Herisanu [20,40] invented this approach for the first time. In addition, they published some papers presented in [19, 25, 26, 27, 39] to show the ability of OHAM to expand their ideas in order to implement it to solve a vast domain of nonlinear problems. The advantage of OHAM is its convergence criteria so that is similar to homotopy analysis method (HAM ) in more flexible area of convergence. Moreover, power of this method has been studied by some researchers $[25,26,19]$. Some different examples are presented to indicate the power of the method compared to HPM in the section of numerical examples. Graphs are used to illustrate solutions obtained by OHAM. Therefore, we have presented a powerful approach OHAM which is generalized form HAM and HPM and has simple procedure to perform .This method has been used by various researchers in different fields as a useful technique [29, 34, 38, 39, 42]. In this paper, we introduce the main structure of OHAM compared to HPM; Three numerical examples are tested and illustrated. At last, we discuss about the obtained computational results. 


\section{Uniqueness and existence theorems in mixed Volterra- Fredholm integral equations}

Now we take into account linear mixed Volterra-Fredholm type integral equation (1) so that $u$ is called an unknown function, $v, f \in C[c, d]$ and $K \in L^{2}[c, d]$. The purpose is to study existence and uniqueness for the solution of equation (1). We apply Banach fixed-point theorem to discuss the existence and uniqueness of physical, biological and applied problems given by the equation (1).

\section{Main Results}

Suppose that $S$ be a space of functions $\phi:[c, d] \rightarrow R^{n}$, continuous in $[c, d]$ and satisfy the condition

$$
|\lambda(x)|=O(\exp (\theta(|x|))), x \in[c, d]
$$

where $\mu>0$ is a constant. In the space $S$, we define the following norm

$$
|\lambda|=\sup _{[c, d]}[|\lambda(x)| \exp (\theta(|x|))]
$$

It is noticed that $S$ is a Banach space. We notice that there is a nonnegative constant $M$ such that

$$
|\lambda(x)| \leq M \exp (\theta(|x|)), x \in[c, d]
$$

Then , we can conclude that

$$
|\lambda| \leq M
$$

Now we give sufficient conditions for the existence and uniqueness of the solutions in equation (1).

\section{Theorem 1:}

We assume that

1) Continuous kernel $K(y, z) v(z)$ defined on $[c, d] \times[c, d]$

such that

$$
\left|K(y, z) v_{1}(z)-K(y, z) v_{2}(z)\right| \leq K(y, z)\left|v_{1}-v_{2}\right|
$$

for $\left(y, z, v_{1}, v_{2}\right) \in[c, d] \times[c, d], i=1,2$ and

$$
\int_{c}^{x} \int_{c}^{d} K(y, z) v(z) \exp (\theta|y|) d y d z \leq Q \exp (\theta|x|)
$$

So that $Q$ is a nonnegative constant.

2) There exists a constant $N>0$ such that

$$
|f(x)|+\int_{a}^{x} \int_{a}^{b} K(y, z) v(z) \exp (\theta|y|) d y d z \leq N \exp (\theta|x|)
$$

If $Q<1$, then we can find $u \in S$ as a solution of Eq.1 that is considered as a limit for sequence of solutions. 


\section{Proof:}

For $v \in S, A(v)$ is defined as an operator in right side of equation (1). It can be shown that $A(v): S \rightarrow S . A(u)$ is continuous in $[c, d]$ and $A(v(x)) \in L^{2}[c, d]$ for $x \in[c, d]$. To verify Eq.2, we have :

$$
\begin{aligned}
& |A(v(x))| \leq \int_{0}^{x} \int_{c}^{d}\left|K\left(y, z, v_{1},()\right)-K\left(y, z, v_{2},()\right)\right| d y d z \\
& +|f(x)|+\int_{0}^{x} \int_{c}^{d} \mid K\left(y, z, v_{2},()|d y d z \leq| v \mid \int_{0}^{x} \int_{c}^{d} h(y, z, v) \exp (\theta|y|) d y d z\right. \\
& +N \exp (|\theta(y)|) d y d z \leq[M Q+N] \exp (\theta|x|) .
\end{aligned}
$$

Therefore, we conclude that $A(u) \in S$. Now we show that the operator $A(u)$ is a contraction mapping.

We assume that $u, z \in S$, and then from the assumption (1), we have

$$
\begin{aligned}
& \|A(v(t, x))-A(z(t, x))\| \\
& \leq \int_{0}^{t} \int_{\Omega}\|F(t, x, s, y, v(s, y))-F(t, x, s, y, z(s, y))\| d y d s \\
& \leq|v-z| \int_{0}^{t} \int_{\Omega} h(t, x, s, y) \exp (\theta(s+\|y\|)) d y d s \\
& \leq Q|v-z| \exp (\theta(t+\|x\|)) \\
& |A(v(x))-A(z(x))| \leq \int_{0}^{x} \int_{c}^{d}|K(y, z, u)-K(y, z, v)| d y d z \\
& \leq|u-v| \int_{0}^{x} \int_{c}^{d} h(y, z, v) \exp (\theta|x|) d y d z \\
& \leq Q|u-v| \exp (\theta|x|)
\end{aligned}
$$

Then , we obtain :

$$
|A(u(x))-A(z(x))| \leq Q|u-v| \exp (\theta|x|)
$$

Therefore, there is a unique solution $u \in S$ of equation (1) and it completes the proof.

\section{Description of Optimal Homotopy Asymptotic Method (OHAM)}

Now, we introduce the general structure of OHAM based on the below scheme. We perform the OHAM to a general nonlinear equation like $A(v(y))=f(y)$ that can be decomposed as

$$
L(v(y))+f(y)+N(v(y))=0
$$

So that $L$ is considered as a linear operator, $N$ an unknown function, $f(y)$ a known function and $N(v(y))$ a non-linear operator. Then, a new homotopy function is made for OHAM as follows [16, 17, 40]:

$$
(1-p)[L(v(y, p))+f(y)]=H(p)[L(v(y, p))+f(y)+N(v(y, p))]
$$


So that $p \in[0,1]$ is a small embedding parameter $(p \neq 0), H(p)$ is an auxiliary function, and $v(y, p)$ is defined as unknown function that needs to be obtained. For values $p=0$ and $p=1$, we have :

$$
v(y, 0)=v_{0}(y), v(y, 1)=v(y)
$$

respectively. When $p$ changes from 0 to 1 , the solution $v(y, p)$ moves from $v_{0}(y)$

to the solution $v(y)$ and $v_{0}(y)$ is attainable from Eq.9 for $p=0$.

$$
L\left[v_{0}(y)\right]+f(y)=0
$$

We can define $H(p)$, the auxilary function as follows :

$$
H(p)=p c_{1}+p^{2} c_{2}+p^{3} c_{3}+\cdots
$$

in which the constants $C_{1}, C_{2}, \ldots$ need to be determined.

Therefore, the solution of Eq.9 can be obtained as

$$
v\left(y ; p, c_{i}\right)=v_{0}(y)+\sum_{k \geq 1} v_{k}\left(y, c_{i}\right) p^{k}, i=1,2, \cdots
$$

Inserting Eq.14 to Eq.10 and finding the coefficients from similer powers of $p, u_{0}(y)$ is obtained by Eq.12.So, we have :

$$
L\left(v_{1}(y)\right)=c_{1} N\left(v_{0}(y)\right)
$$

Therefore, we can obtain general iterative relation as follows:

$$
L\left(v_{k}(y)-v_{k-1}(y)\right)=c_{k} N_{0}\left(v_{0}(y)\right)+\sum_{i=1}^{k-1} c_{i}\left[L\left(v_{k-i}(y)\right)+N_{k-i}\left(v_{0}(y), v_{1}(y), \cdots, v_{k-1}(y)\right)\right]
$$

For the values $k=2,3, \cdots$ such that $N_{m}\left(v_{0}(y), v_{1}(y), \cdots, v_{m}(y)\right)$ can be obtained through using Taylor expansion of $N\left(v, p, c_{i}\right)$ in series with respect to $p$ as follows :

$$
N\left(v\left(y ; p, c_{i}\right)\right)=N_{0}\left(v_{0}(y)\right)+\sum_{m \geq 1} N_{m}\left(v_{0}, v_{1}, \ldots, v_{m}\right) p^{m}
$$

The unknown expression $v\left(y ; p, c_{i}\right)$ is obtained by Eq.14 .The elements $u_{k}$ for $k>0$ are calculated through the system of Eqns.14, 15 and 16 that we can easily solve it. The series Eq.14 converges by obtaining optimal values of auxiliary constants $c_{1}, C_{2}, \ldots, c_{m}$. In $p=1$, it yeilds

$$
v\left(y, c_{i}\right)=v_{0}(y)+\sum_{k \geq 1} v_{k}\left(y, c_{i}\right) .
$$

Then, the solution of Eq.2 is obtained in this form :

$$
v^{m}\left(y, c_{i}\right)=v_{0}(y)+\sum_{k=1}^{m} v_{k}\left(y, c_{i}\right), i=1,2, \ldots, m
$$

Residual is obtained by substituting Eq.19 into Eq.9 as follows : 


$$
R\left(y ; c_{i}\right)=L\left(v^{m}\left(y, c_{i}\right)\right)+g(y)+N\left(v^{m}\left(y, c_{i}\right)\right), \quad i=1,2, \ldots, m
$$

If $R\left(y ; c_{i}\right)=0$, then $u^{m}\left(y, c_{i}\right)$ is the exact solution.Auxiliary constants $c_{i}, i=1,2, \ldots$ can be obtained by finite element methods like Galerkin, Ritz,Collocation and Least Squares methods. Now, we use least square method to minimize the residual function.Then, we have :

$$
J\left(c_{i}\right)=\int_{c}^{d} R^{2}\left(y, c_{i}\right) d y,
$$

The values $C$ and $d$ are optional and can be chosen based on the type of problem and its given interval . By forming system of normal equations, we find optimal parameters $c_{i}, i=1,2, \ldots, m$ as follows :

$$
\frac{\partial J}{\partial c_{1}}=\frac{\partial J}{\partial c_{2}}=\cdots=\frac{\partial J}{\partial c_{m}}=0 .
$$

Therefore, the favorite approximate solution is easily obtained. Then, if $k \in[c, d]$, putting $k_{i}$ into Eq.20 gives the follwing equation :

$$
R\left(k_{1}, c_{i}\right)=R\left(k_{2}, c_{i}\right)=\cdots=R\left(k_{m}, c_{i}\right), \quad i=1,2, \cdots, m
$$

Therefore, we can propose advantages and disadvantages of the method as follows:

(1) OHAM sometimes takes a lot of time to evaluate the residual by increasing the convergence parameters. Therefore, computing of convergence constants is not feasible over three or four times. Timeconsuming problems have presented by [33] and [41, 46].

(2) Although OHAM obtains best approximations for many cases but the closed form solution cannot be achieved all the time. This case occurs due to the existence of the convergence constants $C_{i}$ in the auxiliary function $H(p)$.

\section{Structure of Homotopy Perturbation Method(HPM)}

To express this method, we make homotopy function by a small embedding parameter $p \in[0,1]$, . The structure of method includes a deformation from intial solution to the final step that is obtained our favorite solution. This method has been used by many researchers to solve different types of non linear problems[9, 10,14,21,22,23,34,35,12]. HPM is considered as a combination of the classical perturbation technique and the homotopy from topology in pure mathematics It is not restricted to small parameters like traditional perturbation methods.We can find highly accurate solutions in just few iterations. He in [23] extended methods of nonlinear analysis for a vast range of problems. In order to explain the structure of HPM, we assume the following nonlinear functional equation

$$
A(v)-f(y)=0, y \in \Omega
$$

with the following boundary conditions:

$$
B\left(v, \frac{\partial v}{\partial y}\right)=0, y \in \Gamma
$$


So that $A$ is considered as general differential operator, $B$ a boundary operator, $f(y)$ a known analytic function, and $\Gamma$ the domain boundary for $A$ can be divided into two operators $L$ and $N$, such that $L$ is linear and $N$ is nonlinear so that Eq.24 can be cosidered as

$$
L(v)+N(v)-f(y)=0 .
$$

Now, we construct the homotopy function as :

$$
\begin{aligned}
& H(V, p)=(1-p)\left[L(V)-L\left(v_{0}\right)\right]+ \\
& p[L(v)+N(v)-f(y)]=0, p \in[0,1], y \in \Omega
\end{aligned}
$$

or

$$
\begin{aligned}
& H(V, p)=L(V)-L\left(v_{0}\right)+ \\
& p[L(v)+N(v)-f(y)]=0, p \in[0,1], y \in \Omega
\end{aligned}
$$

Such that $p$ is in the range of zero and one as homotopy parameter and $v_{0}$ is the first approximation for the solution of Eq.24 that satisfie in the boundary conditions. Eq.24 or Eq.26 is written as a power series of $p$

$$
V=v_{0}+p v_{1}+p^{2} v_{2}+\cdots
$$

Substituting Eq.29 into Eq.27 or Eq.28 and equating similar powers of $p$, we can obtain the sequence $v_{0}, v_{1}, v_{2}, \ldots$.When $p \rightarrow 1$, the approximate solution is given for Eq.24 in the form

$$
V=v_{0}+v_{1}+v_{2}+v_{3}+\cdots
$$

\section{Convergence theorem for optimal homotopy asymptotic method:}

If the series

$$
v\left(y, c_{i}\right)=v_{0}(y)+\sum_{k \geq 1} v_{k}\left(y, c_{i}\right)
$$

converges to $v(y)$ where $v_{k}(y) \in L^{2}[c, d]$ in which it can be produced by relations:

$$
\begin{gathered}
L\left(v_{1}(y)\right)=c_{1} N\left(v_{0}(y)\right) \\
L\left(v_{k}(y)-v_{k-1}(y)\right)=c_{k} N_{0}\left(v_{0}(y)\right)+\sum_{i=1}^{k-1} c_{i}\left[L\left(v_{k-i}(y)\right)+N_{k-i}\left(v_{0}(y), v_{1}(y), \cdots, v_{k-1}(y)\right)\right]
\end{gathered}
$$

Such that $k=2,3, \cdots$.Then, $v(y)$ is the exact solution of the problem as follows:

$$
L(v(y))+f(y)+N(v(y))=0
$$

Proof: If the series

$$
\sum_{k \geq 1}^{\infty} v_{k}\left(y, c_{i}\right)
$$

converges, it can be written as

$$
S(x)=\sum_{k=1}^{\infty} v_{k}\left(y, c_{i}\right)
$$


In addition, it holds that

$$
\lim _{k \rightarrow \infty} v_{k}\left(y, c_{i}\right)=0
$$

Left hand side of relation (16) satisfies

$$
v_{1}\left(y, c_{1}\right)+\sum_{k=2}^{n} v_{k}\left(y, c_{k}\right)-\sum_{k=2}^{n} v_{k-1}\left(y, c_{k-1}\right)=v_{2}\left(y, c_{2}\right)-v_{1}\left(y, c_{1}\right)+v_{n}\left(y, c_{n}\right)-v_{n-1}\left(y, c_{n-1}\right)=v_{n}\left(y, c_{n}\right)
$$

According to summation relation in (36), we have:

$$
v_{1}\left(y, c_{1}\right)+\sum_{k=2}^{n} v_{k}\left(y, c_{k}\right)-\sum_{k=2}^{n} v_{k-1}\left(y, c_{k-1}\right)=\lim _{n \rightarrow \infty} v_{n}\left(y, c_{n}\right)=0
$$

By using linear operator $L$, we have:

$$
\begin{aligned}
& L\left(v_{1}\left(y, c_{1}\right)\right)+\sum_{k=2}^{\infty} L\left(v_{k}\left(y, c_{k}\right)\right)-\sum_{k=2}^{\infty} L\left(v_{k-1}\left(y, c_{k-1}\right)\right) \\
& =L\left(v_{1}\left(y, c_{1}\right)\right)+L\left(\sum_{k=2}^{\infty} L\left(v_{k}\left(y, c_{k}\right)\right)\right)-L\left(\sum_{k=2}^{\infty} L\left(v_{k-1}\left(y, c_{k-1}\right)\right)\right)=0
\end{aligned}
$$

that satisfies in the relation:

$$
\begin{aligned}
& L\left(v_{1}\left(y, c_{1}\right)\right)+L\left(\sum_{k=2}^{\infty} v_{k}\left(y, c_{k}\right)\right)-\left(\sum_{k=2}^{\infty} v_{k-1}\left(y, c_{k-1}\right)\right) \\
& =\sum_{k=2}^{\infty}\left[c_{k} N_{0}\left(v_{0}(y)\right)+\sum_{i=1}^{k-1} c_{i} L\left(v_{k-i}\left(y, c_{k-i}\right)+N_{k-i}\left(v_{k-i}\left(y, c_{k-1}\right)+f(y)\right]=0\right.\right.
\end{aligned}
$$

Right hand side of Eq.41 can be written as follows:

$$
\sum_{k=1}^{\infty}\left[\sum_{i=1}^{k} c_{i-k}\left[L\left(v_{i-1}\left(y, c_{i-k}\right)\right)+N_{i-1}\left(v_{k-1}, c_{k-1}\right)\right]\right]+f(y)=0
$$

If $c_{i}, i=1,2, \cdots, m$ are chosen appropriately, then relation (42) leads to

$$
L(v(y))+f(y)+N(v(y))=0
$$

That is the exact solution of the problem.

\section{Illustrative examples}

7.1. Example1. We consider the mixed Volterra-Fredholm integral equation as follows:

$$
v(x)=11 x+\frac{17}{2} x^{2}+\int_{0}^{x} \int_{0}^{1}(y-z) v(z) d z d y .
$$

in which whose exact solution is given by $v(x)=6 x+12 x^{2}$.

Item1: Homotopy Perturbation method (HPM)

The Homotopy function can be constructed from Eq.44 as follows:

$$
H(v, p)=v(x)-f(x)-p \int_{0}^{x} \int_{0}^{1}(y-z) v(z) d z d y
$$


where $f(x)=11 x+\frac{17}{2} x^{2}$. By using Eq.29 into Eq.45 and finding the coefficients from similar powers of $p$, we have :

$$
\begin{aligned}
& v_{0}(x)=f(x)=11 x+\frac{17}{2} x^{2} \\
& v_{i}(x)=\int_{0}^{x} \int_{0}^{1}(y-z) v_{i}(z) d z d y
\end{aligned}
$$

In this case, our experience shows that in 16-th iteration we can obtain the approximate solution as follows:

$$
\tilde{u}(x)=\sum_{i=0}^{16} u_{i}(x)=\frac{(x(2218611106740437146+4437222213480873833 x))}{369768517790072832}
$$

The comparative graph of approximate and exact solution of Example1 by HPM and graph of error function are illustrated in Fig.1 and Fig.2. In addition, numerical results have been tabulated in Table 1.

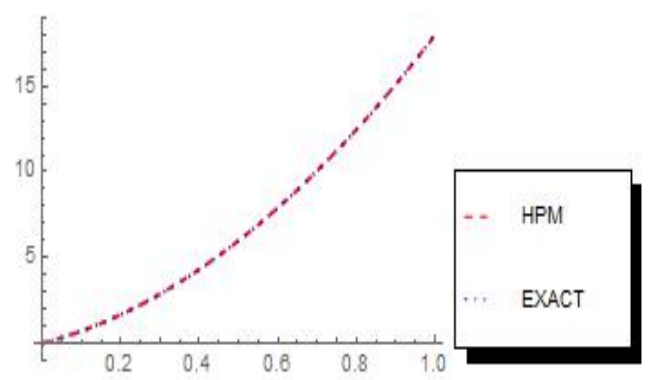

Fig1. Graph of approximate and exact solutions of Example 1 by HPM.

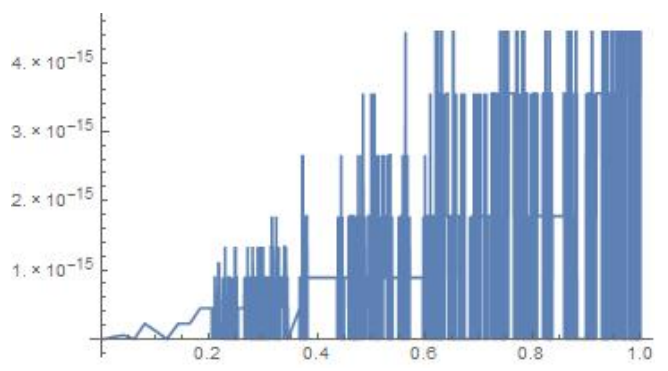

Fig2. The error function of Example 1 by HPM.

Item2: Optimal Homotopy Asymptotic method (OHAM)

We construct homotopy function from Eq.44 as follows:

$$
\begin{aligned}
& f(x)=11 x+\frac{17}{2} x^{2} \\
& H(v, p)=(1-p)(v(x)-f(x))=H(p)\left(v(x)-f(x)-\int_{0}^{x} \int_{0}^{1}(y-z) v(z) d z d y\right)
\end{aligned}
$$

By using Eq.29 into Eq.47 and doing the similar procedures, we have :

$$
v_{0}(x)=11 x+\frac{17}{2} x^{2}
$$




$$
\begin{aligned}
& v_{1}(x)=-\frac{1}{24} x(-139+100 x) c_{1} \\
& v_{2}(x)=\frac{1}{288}\left(\begin{array}{l}
1668 x c_{1}-1200 x^{2} c_{1}+1924 x c_{1}^{2} \\
-1417 x^{2} c_{1}^{2}+1668 x c_{2}-1200 x^{2} c_{2}
\end{array}\right)
\end{aligned}
$$

Then, by using least square method presented in section 3 , we find real optimal parameters $C_{1}$ and $C_{2}$ among a set of complex and real roots as follows:

$$
c_{1}=0.923077, c_{2}=-3.69231
$$

Then, the series solution is given as:

$$
v(x)=11 x+\frac{17}{2} x^{2}-0.038461538461538464 x(-139+100 x)+\frac{1}{288}\left(\begin{array}{l}
-2979.692307692308 x \\
+2115.692307692308 x^{2}
\end{array}\right)
$$

The comparative graph of approximate and exact solution of Example 1 by OHAM and graph of error function are illustrated in Figure 3 and Figure 4.

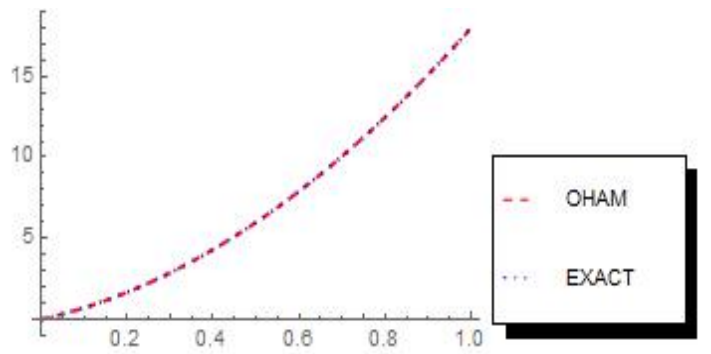

Fig3. Graph of approximate and exact solutions of Example 1 by OHAM.

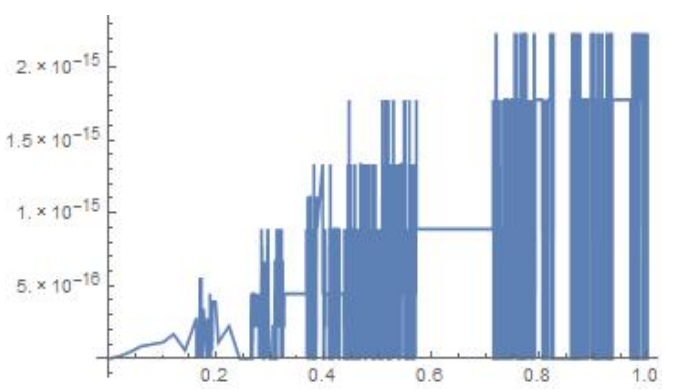

Fig4. The error function of Example 1 by OHAM.

\begin{tabular}{llll}
\multicolumn{3}{c}{ Table1. Approximate and exact solutions with absolute error of Example 1 by OHAM. } \\
\hline$X$ & OHAM & Exact solution & Absolute Error \\
0 & 0 & 0 & 0 \\
0.1 & 0.720000 & 0.720000 & 0 \\
0.2 & 1.680000 & 1.680000 & 0 \\
0.3 & 2.880000 & 2.880000 & 0 \\
0.4 & 4.320090 & 4.320000 & 0 \\
0.5 & 6.000000 & 6.000000 & 0 \\
0.6 & 7.920000 & 7.920000 & 0 \\
0.7 & 10.080000 & 10.080000 & 0 \\
\hline
\end{tabular}




\begin{tabular}{llll}
\hline 0.8 & 12.480000 & 12.480000 & 0 \\
0.9 & 15.120000 & 15.120000 & 0 \\
1.0 & 18.000000 & 18.000000 & 0 \\
\hline
\end{tabular}

7.2. Example2. We consider the mixed Volterra-Fredholm integral equation as follows:

$$
v(x)=2+4 x-\frac{9}{8} x^{2}-5 x^{3}+\int_{0}^{x} \int_{0}^{1}(y-z) v(z) d z d y .
$$

In which the exact solution is given by $v(x)=2+3 x-5 x^{3}$.

Item1: Homotopy Perturbation method (HPM)

The Homotopy function can be constructed from Eq.28 as follows:

$$
H(v, p)=(1-p)(v(x)-f(x))=v(x)-f(x)-p \int_{0}^{x} \int_{0}^{1}(y-z) v(z) d z d y
$$

where $f(x)=2+4 x-\frac{9}{8} x^{2}-5 x^{3}$

By using Eq.29 into Eq.50 and finding the coefficients from similar powers of $p$, we obtain solutions similar to Eq.46 with $v_{0}(x)=f(x)=2+4 x-\frac{9}{8} x^{2}-5 x^{3}$.

The comparative graph of approximate and exact solution of Example 2 by HPM and graph of error function are illustrated in Fig.5 and Fig.6. It can be shown that increasing the number of iterations cannot effect on the accuracy of approximation obtained by HPM. This shows that this method has been saturated. Therefore, HPM has disability to solve this integral equation. Partial fractions are provided in higher iterations and continuing the iterations is not effective.

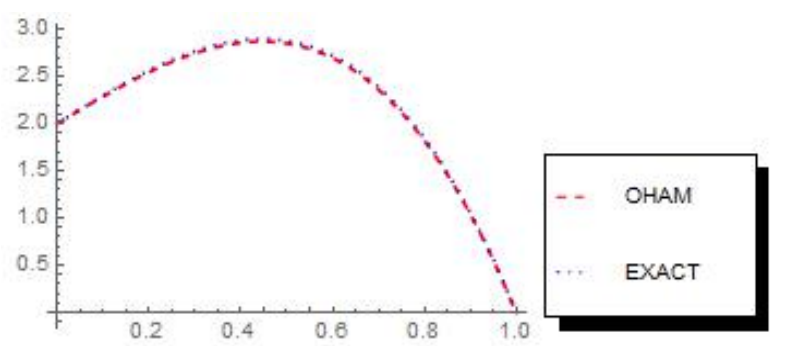

Fig5. Graph of approximate and exact solutions of Example 2 by HPM.

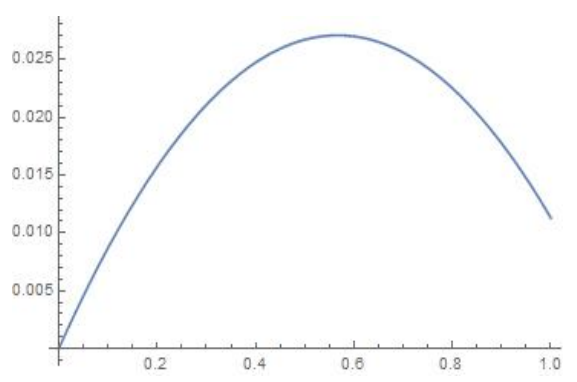

Fig6. The error function of Example 2 by HPM. 
Item 2: Optimal Homotopy Asymptotic method (OHAM)

The homotopy function for Eq.28 is made as follows:

$$
\begin{aligned}
& f(x)=2+4 x-\frac{9}{8} x^{2}-5 x^{3} \\
& H(v, p)=(1-p)(v(x)-f(x))=H(p)\left(v(x)-f(x)-\int_{0}^{x} \int_{0}^{1}(y-z) v(z) d z d y\right)
\end{aligned}
$$

By using Eq.22 into Eq.51 and finding the coefficients from similar powers of $p$, we have :

$$
\begin{aligned}
& v_{0}(x)=2+4 x-\frac{9}{8} x^{2}-5 x^{3} \\
& v_{1}(x)=-\frac{1}{96} x(-101+114 x) c_{1} \\
& v_{2}(x)=\frac{1212 x c_{1}-1368 x^{2} c_{1}+1274 x c_{1}^{2}}{1152}+\frac{-1443 x^{2} c_{1}^{2}+1212 x c_{2}-1368 x^{2} c_{2}}{1152}
\end{aligned}
$$

Moreover, by using least square method presented in section 2, we find real optimal parameters $C_{1}$ and $C_{2}$ among a set of complex and real roots as follows:

$$
c_{1}=0.923077, c_{2}=-3.69231
$$

Then, we obtain the series solution as

$$
\begin{aligned}
& v(x)=2+4 x-\frac{9}{8} x^{2}-5 x^{3}-0.009615384615384616 x(-101+114 x) \\
& +\frac{-2270.769230769231 x}{1152}+\frac{+2558.7692307692314 x^{2}}{1152}
\end{aligned}
$$

The comparative graph of approximate and exact solution of Example 2 and graph of error function are illustrated in Fig.7 and Fig.8.Also; numerical results have been tabulated in Table 2.

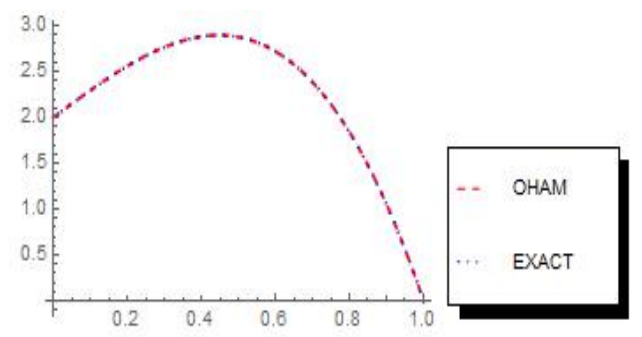

Fig7. Graph of approximate and exact solutions of Example 2 by OHAM. 


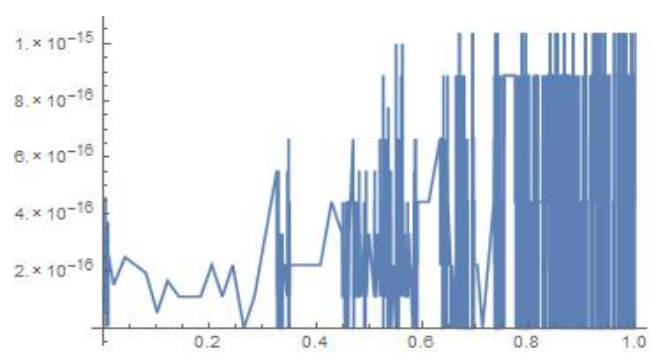

Fig8. The error function of Example 2 by OHAM.

\begin{tabular}{lccc}
\multicolumn{2}{c}{ Table2. Approximate and exact solutions with absolute error of Example 2 by OHAM. } \\
\hline$X$ & OHAM & Exact solution & Absolute Error \\
0 & 2.000000 & 2.000000 & 0 \\
0.1 & 2.295000 & 2.295000 & 0 \\
0.2 & 2.560000 & 2.560000 & 0 \\
0.3 & 2.765000 & 2.765000 & 0 \\
0.4 & 2.880000 & 2.880000 & 0 \\
0.5 & 2.875000 & 2.875000 & 0 \\
0.6 & 2.720000 & 2.720000 & 0 \\
0.7 & 2.385000 & 2.385000 & 0 \\
0.8 & 1.840000 & 1.840000 & 0 \\
0.9 & 1.055000 & 1.055000 & 0 \\
1.0 & 0.000000 & 0.000000 & 0
\end{tabular}

7.3. Example3. The mixed Volterra-Fredholm integral equation is defined as follows:

$$
v(x)=\cos x+\sin x-x^{2}+\frac{\pi}{2} x+\int_{0}^{x} \int_{0}^{1}(y-z) v(z) d z d y .
$$

In which whose exact solution is given by $v(x)=\cos x+\sin x$.

Item1: Homotopy Perturbation method (HPM)

The Homotopy function can be constructed from Eq.53 as follows:

$$
H(v, p)=v(x)-f(x)-p \int_{0}^{x} \int_{0}^{1}(y-z) v(z) d z d y
$$

where $f(x)=\cos x+\sin x-x^{2}+\frac{\pi}{2} x$.

By using Eq.22 into Eq.54 and finding the coefficients from similar powers of $p$, we have a sequence of solutions similar to Eq.46 with $v_{0}(x)=f(x)=\cos x+\sin x-x^{2}+\frac{\pi}{2} x$.

Again, in this case, there is no convergent series solution in close agreement with exact solution and increasing the number of iterations is ineffective too.

Item 2: Optimal Homotopy Asymptotic method (OHAM)

We construct homotopy function from Eq.2.2 as follows:

$$
\begin{aligned}
& f(x)=\cos x+\sin x-x^{2}+\frac{\pi}{2} x \\
& H(v, p)=(1-p)(v(x)-f(x))=H(p)\left(v(x)-f(x)-\int_{0}^{x} \int_{0}^{1}(y-z) v(z) d z d y\right)
\end{aligned}
$$


By using Eq.22 into Eq.55 and finding the coefficients from similar powers of $p$, we obtain :

$$
\begin{aligned}
& v_{0}(x)=f(x)=\cos x+\sin x-x^{2}+\frac{\pi}{2} x \\
& v_{1}(x)=\frac{1}{192}\left(96+\pi^{3}\right)(\pi-2 x) x c_{1}
\end{aligned}
$$

In addition, by using least square method presented in section 2, we find real optimal parameter $C_{1}$ as follows:

$$
c_{1}=-0.755868
$$

Therefore, the approximate solution is given by:

$$
v(x)=\frac{\pi x}{2}-0.5(\pi-2 x) x-x^{2}+\cos x+\sin x
$$

Graph of approximate and exact solutions of Example 4 and its error function are illustrated in Fig.9 and Fig.10. In addition, numerical results have been tabulated in Table 3.

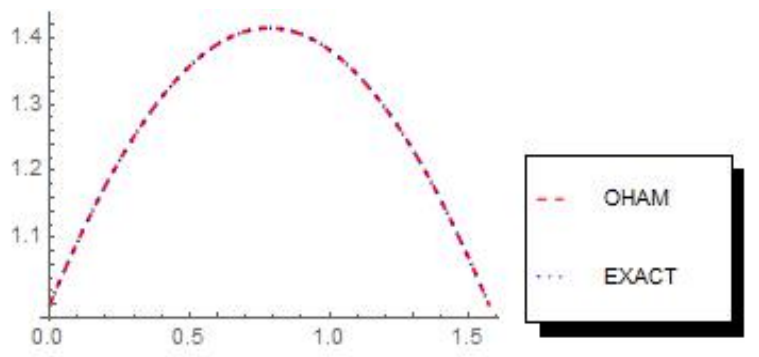

Fig9. Graph of approximate and exact solutions of Example 4 by OHAM.

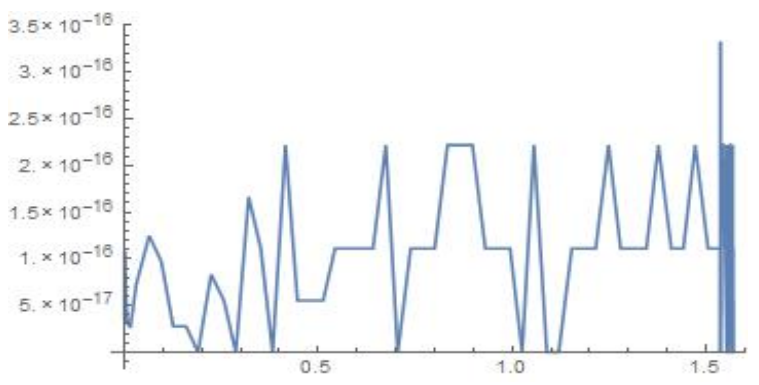

Fig10. The error function of Example 4 by OHAM.

Table3. Approximate and exact solutions with absolute error of Example 3 by OHAM.

\begin{tabular}{llll}
\hline$X$ & OHAM & Exact solution & Absolute Error \\
0 & 1.000000 & 1.000000 & 0 \\
$\pi / 20$ & 1.144120 & 1.144120 & 0 \\
$2 \pi / 20$ & 1.260070 & 1.260070 & 0 \\
$3 \pi / 20$ & 1.345000 & 1.345000 & 0 \\
$4 \pi / 20$ & 1.396800 & 1.396800 & 0 \\
$5 \pi / 20$ & 1.414210 & 1.414210 & 0 \\
$6 \pi / 20$ & 1.396800 & 1.396800 & 0 \\
$7 \pi / 20$ & 1.345000 & 1.345000 & 0 \\
$8 \pi / 20$ & 1.260070 & 1.260070 & 0 \\
\hline
\end{tabular}




\begin{tabular}{llll}
\hline $9 \pi / 20$ & 1.144120 & 1.144120 & 0 \\
$\pi / 2$ & 1.000000 & 1.000000 & 0
\end{tabular}

\section{Results and discussion}

The purpose of the present paper is to demonstrate the power of OHAM with respect to HPM in order to find the solutions of mixed Volterra-Fredholm integral equations. We implemented all computations in a laptop by processor $2.53 \mathrm{GHz}$ and we could handle them in OHAM until suitable number of iterations. Therefore, we confront with high cost of computations that occupy a vast space of our memory and it is natural that the process of computations takes a long time especially to compute integral components directly. If we have powerful processors, we can enhance accuracy of the OHAM approximations. Thus, we can conclude that the obtained results of OHAM method can solve mixed Volterra-Fredholm integral equations in just few iterations rather than HPM in high number of iteration and make favorite approximations close to exact solutions. It means that we can find a suitable series solution close to exact solution of expressed examples if we continue to computations in just enough number of iterations. In spite of the cost of computations, the OHAM method is very powerful, reliable, efficient and accurate compared to HPM and many competitive numerical methods that sometimes equations need to be changed before solving the problem by some approaches such as change of variables, Laplace transform, and mesh-based methods by a huge cost of programming and so on. Therefore, we can perform OHAM directly on any favorite problem without any concern. In addition, in cases that we have complicated integrals, quadrature methods such as Trapezoidal and Simpson rules can be used to approximate integral components numerically and it cannot make a problem in general to solve integral equations by implementation of OHAM and HPM.

\section{Conclusions}

In this paper, OHAM and HPM were employed to solve Mixed Volterra-Fredholm integral equations of the second kind. Our approach is time independent. In addition, our comparison shows that OHAM is a powerful method to solve these types of integral equations and we found out that HPM has powerlessness to solve these types of equations for high successive iterations to find an accurate approximation. The Advantage of OHAM with respect to HPM is optimal parameters $C_{i}$. In fact; these parameters play an important role to find approximations with high accuracy. In addition, other types of mixed integral equations can be solved easily by means of OHAM.

\section{Acknowledgment}

Authors would like to thank the reviewers for their helpful suggestions and assistance with this research paper. In addition, authors would like to appreciate for supports of Islamic Azad UniversityCentral Tehran Branch in order to assign educational facilities.

\section{References}


[1] S.S. Ahmed, Expansion Method for Solving Fredholm Integral Equations in Space-Time, Journal of Kirkuk University, 2006, Vol.1, No.1.

[2] F. Bloom, Asymptotic bounds for solutions to a system of damped integro-differential equations of electromagnetic theory, J. Math. Appl. 73 (1980) 524-542.

[3] H. Brunner, J.P. Kautheu, The Numerical Solution of Two-Dimensional Volterra Integral Equations by Collocation and Iterated Collocation. IMA Journal of Numerical Analysis, 1989, 9, 47-59.

[4] H.Brunner, On the numerical solution of nonlinear Volterra-Fredholm integral equations by collocation methods, SIAM J. NUMER. ANAL.Vol. 27, No. 4, 1990, pp. 987-1000.

[5] J.P. Chilues, and P.Delfiner, Geostatistics: Modeling Spatial Uncertainty. John Wiley, New York (1999).

[6] N. Cressie, Statistics for Spatial Data. Second edition, John Wiley, New York, (1993).

[7] N. Cressie and H.-C. Huang, Classes of nonseparable, spatio-temporal stationary co- variance functions. J. Amer. Statist. Assoc. 94, (1999), 1330-1340.

[8] R.Y. Chang, M.L. Wang, Shifted Legendre direct method for variational problems, J. Optim. Theory Appl. 39 (1983) 229-307.

[9] M. Dehghan, and F. Shakeri, Approximate solution of a differential equation arising in astrophysics using the variational iteration method, New Astronomy, 2008,13,53-59

[10] M. Dehghan and F. Shakeri ,Solution of an Integro-Differential equation arising in oscillating Magnetic fields using He’s Homotopy Perturbation Method, PIER, 2008,78,361-376.

[11] O. Diekmann, Thresholds and traveling for the geographical spread of infection, J. Math. Biol. 6 (1978) 109-130.

[12] Uriel Filobello-Nino et.al, A handy approximate solution for a squeezing flow between two infinite plates by using of Laplace transform-homotopy perturbation method, SpringerPlus 2014, 3:421.

[13] L.K. Forbes, S.Crozier, D.M. Doddrell, Calculating current densities and fields produced by shielded magnetic resonance imaging problems, SIAM J. Appl. Math. 57 (1997) 401-425.

[14] D.D. Ganji and A.Rajabi, Assessment of Homotopy Perturbation and Perturbation Methods in heat radiation equations, Int.Commun. Heat Mass Transfer, 2006, 33,391-400.

[15] C. H. Hasiao, Hybrid function method for solving Fredholm and Volterra integral equations of the second kind, J. Comput. Appl. Math. 230 (2009) 59-68.

[16] M.S. Hashmi, N. Khan, S. Iqbal, Optimal homotopy asymptotic method for solving nonlinear Fredholm integral equations of second kind, Applied Mathematics and Computation, 218: 10982-10989 (2012)

[17] M.S. Hashmi, N. Khan, S. Iqbal, Numerical Solutions of Weakly Singular Volterra Integral Equations Using Optimal Homotopy Asymptotic Method, Computers and Mathematics with Applications, 64: 1567-1574 (2012)

[18] Handcock, M.S. and Wallis, J.R. (1994), An approach to statistical spatial-temporal modeling of meteorological fields (with discussion). J. Amer. Statist. Assoc. 89, 368-390. 
[19] S. Haq, M. Idrees ,S. Islam, Application of optimal homotopy asymptotic method to eight order initial and boundary value problems,I.J.Appl.Math.Comput,2010,2(4) 73-80.

[20] N. Herisanu, V. Marinca, T. Dordea, and G. Madescu, A new analytical approach to nonlinear vibration of an Electrical machine, Proceedings of the Romanian Academy. Series A, 2008, vol.9, no.3,229-236.

[21] J.H.He, Homotopy perturbation technique, Comput. Methods Appl.Mech.Engrg,1999, 178,257262.

[22] J.H.He, An approximate solution technique depending upon an artificial parameter, Commun. Nonlinear Sci. Simul, 1998, 3(2), 92-97.

[23] J.H.He, A coupling method of homotopy technique and perturbation technique for nonlinear problems, Int. J. Nonlinear Mech, 2000,35, 37-43.

[24] K.Holmaker, Global asymptotic stability for a stationary solution of a system of integrodifferential equations describing the formation of liver zones, SIAM J. Math. Anal. 24 (2003), 116-128.

[25] S. Iqbal, M. Idrees, A.M. Siddiqui, A.R. Ansari, Some solutions of the linear and nonlinear Klein-Gordon equations using the optimal homotopy asymptotic method, Appl. Math.Compu, 2010, 216(10), 2898-2909.

[26] S. Iqbal ,A. Javed ,Application of optimal homotopy asymptotic method for the analytic solution of singular Lane-Emden type equation ,Appl.Math.Comput,2011,217(19),7753-7761.

[27] M. Javidi, A. Golbabai, Modified homotopy perturbation method for solving non-linear Fredholm integral equations, Chaos Solit. Fract. 40, (2009), 1408-1412.

[28] A.J. Jerri, Introduction to Integral Equations with Applications, second ed., John Wiley and Sons, 1999.

[29] N. Khan, T. Mahmood, M.S. Hashmi, OHAM solution for thin film flow of a third order fluid through porous medium over an inclined plane, Heat Transfer Res. 44 (8),(2013) 113.

[30] N. Khan, M.S. Hashmi, S. Iqbal, T. Mahmood, Optimal homotopy asymptotic method for solving Volterra integral equation of first kind, Alexandria Engineering Journal, (2014), 53(3): 751-755.

[31] E. V. Kovalonko, Some approximate methods for solving integral equations of mixed problems, Provl.Math. Mech. 53, (1989), 85-92.

[32] H. Lechoslaw, Computational results for integral equations in Space-Time, Computational Methods in Science and Technology, 2002, 8(1), 7-15.

[33] S.J. Liao, An optimal homotopy-analysis approach for strongly nonlinear differential equations, Communication in Nonlinear Science and Numerical Simulation 2009;doi:10.1016/j.cnsns.2009 .09 .002 .

[34] S.J. Liao, An approximate solution technique not depending on small parameters: A special examples, Int. J. Nonlinear Mech, 1995, 30(3), 371-380.

[35] S.J. Liao . Boundary element method for general nonlinear differential operators, Eng. Anal. Boundary Elements, 1997, 20, 91-98. 
[36] Y. Mahmoudi, Wavelet Galerkin method for numerical solution of nonlinear integral equation, Appl. Math. Comput, 167, (2005), 1119-1129.

[37] K.V. Mardia, and Goodall, C.R., Spatial-temporal analysis of multivariate environmental monitoring data. In Multivariate Environmental Statistics, eds. G.P. Patil and C.R. Rao, Elsevier Science Publishers, (1993), pp. 347-386.

[38] V. Marinca , N. Herisanu, Determination of periodic solutions for the motion of a particle on a rotating parabola by means of the optimal homotopy asymptotic method, J. Sound Vib,329,(2010), pp.1450-1459.

[39] V. Marinca, N. Herisanu, The optimal homotopy asymptotic method for solving Blasius equation, Appl. Maths. Comput. 231, (2014), 134-139.

[40] V. Marinca and N. Herisanu, Application of optimal homotopy asymptotic method for solving nonlinear equations arising in heat transfer, Int. Commun. Heat Mass Transfer, vol.35, No.6, (2008), 710-715.

[41] Z. Niu, C. Wang, one-step optimal homotopy analysis method for nonlinear differential equations. Communication in Nonlinear Science and Numerical Simulation. Online.doi:10.1016/j.cnsns.2009.08.014.

[42] R.A. Shah, S. Islam and A. M. Siddiqui, Wire coating analysis with Oldroyd 9-constant fluid by Optimal Homotopy Asymptotic Method ,Comp. Math. Appl. 63, (2012), pp. 695-707.

[43] H.R. Thieme, A model for the spatial spread of an epidemic, J. Math. Biol., 4 (1977), pp. 337351.

[44] C. Vasile, Numerical Solution of Two-dimensional non-Linear Fredholm Integral Equations of the Second Kind by Splint Functions. General Mathematics, 2001, Vol.9, No.1.

[45] A.M. Wazwaz, A reliable treatment for mixed Volterra-Fredholm integral equations, Appl. Math. Comput., 127 (2002) 405-414.

[46] C. Wang, Z. Niu, Reply to Comments on A one-step optimal homotopy analysis method for nonlinear differential equations, Communication in Nonlinear Science and Numerical. Simulation. doi: 10.1016/j.cnsns.2009.12.026.

[47] S. Yalcinbas, M. Sezer, The approximate solution of high-order linear Volterra-Fredholm integro-differential equations in terms of Taylor polynomials, Appl .Math .Comput, 2000, 112:291-308.

[48] S. Yalcinbas, Taylor polynomial solutions of nonlinear Volterra-Fredholm integral equations, Appl.Math.Com, Vol.127, 2002, pp 195-206.

[49] S.A. Yousefi, A. Lotfi, M.Dehghan, He's variational iteration method for solving nonlinear mixed Volterra-Fredholm integral equations, Computers and Mathematics with Applications 58 (2009) 2172_2176. 